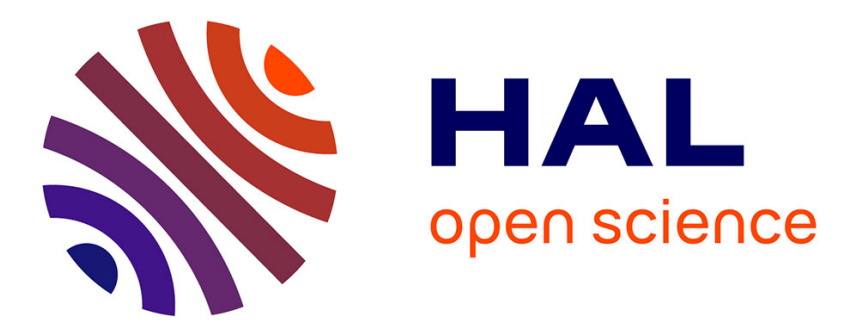

\title{
Spectroscopy of Atoms Confined to Micrometric Pores in Glass
}

\author{
H Failache, A Amy, S Villalba, L Lenci, A Laliotis, A Lezama
}

\section{To cite this version:}

H Failache, A Amy, S Villalba, L Lenci, A Laliotis, et al.. Spectroscopy of Atoms Confined to Micrometric Pores in Glass. OSA Latin America Optics \& Photonics Conference (LAOP 2016), Aug 2016, Medellin, Colombia. 10.1364/LAOP.2016.LTu2B.5 . hal-01399960

\section{HAL Id: hal-01399960 https://hal.science/hal-01399960}

Submitted on 21 Nov 2016

HAL is a multi-disciplinary open access archive for the deposit and dissemination of scientific research documents, whether they are published or not. The documents may come from teaching and research institutions in France or abroad, or from public or private research centers.
L'archive ouverte pluridisciplinaire HAL, est destinée au dépôt et à la diffusion de documents scientifiques de niveau recherche, publiés ou non, émanant des établissements d'enseignement et de recherche français ou étrangers, des laboratoires publics ou privés. 


\title{
Spectroscopy of atoms confined to micrometric pores in glass
}

\author{
H. Failache ${ }^{1}$, L. Amy ${ }^{1}$, S. Villalba ${ }^{1}$, L. Lenci ${ }^{1}$, A. Laliotis ${ }^{2}$, A. Lezama ${ }^{1}$ \\ ${ }^{1}$ Instituto de Física, Facultad de Ingeniería, Universidad de la República, \\ J. Herrera y Reissig 565, 11300 Montevideo, Uruguay \\ ${ }^{2}$ Laboratoire de Physique des Lasers UMR 7538 du CNRS, Université Paris-13, Sorbonne Paris Cité F-93430, \\ Villetaneuse, France \\ heraclio@fing.edu.uy
}

\begin{abstract}
We present two-photon spectroscopy of rubidium vapor contained in the interstitial cavities of porous glass. We report on the first observation of Rydberg atoms under 3D spatial confinement in these micrometric size dielectric cavities.
\end{abstract}

OCIS codes: $300.6210,020.6580$.

\section{Introduction}

With the initial motivation of studying atomic vapors under a 3D spatial confinement, during the last few years we have been studying atomic spectroscopy of alkali atoms in the micrometric interstices of porous glass. Porous glass is a highly scattering medium for light which can appear as detrimental for many applications. However, randomness and disorder could be sometimes a welcome property for some applications as random lasers or alignment insensitive spectroscopical references [2].

The light field interacting with atoms contained in pores deep inside the sample has a speckle structure in which the polarization, amplitude and phase have rapid fluctuations over distances of the order of one wavelength. Due to the atomic thermal velocity, an atom explores large variations of the light field during its interaction time with the light. As a consequence of this motion induced field averaging, coherence effects are difficult to observe in the porous samples while unusual spectral lineshapes are observed for incoherent processes.

The transmission spectrum of $\mathrm{Rb}$ contained in the porous glass is unusual since for low enough atomic density and low confinement, the expected $\mathrm{Rb}$ absorption lines are highly suppressed. This is a consequence of the diffusive propagation of light that makes indistinguishable the detection of photons directly coming from the laser from those emitted as fluorescence. However, when the atomic vapor is spatially confined, the probability for an excited atom to collide with the dielectric surface and decay non-radiativelly is not negligible. Thus confinement favors a non-radiative de-excitation channel that restores the absorption lines in the transmission spectrum [1]. Moreover, at higher atomic densities, radiation trapping increases the probability to excite an atom close to a surface and consequently the probability for non-radiative de-excitation.

Different spectral structures are observed in the light back-scattered by the porous sample in the direction of the incident laser beam. Sub-Doppler saturated absorption resonances are observed. In this configuration the incoming beam saturates the atomic medium and the back-scattered field acts as a probe field. The sub-Doppler resonances width are essentially determined by the probe wave-vector dispersion which is mainly determined by detection solid angle [2]. This experiment shows that, as would be expected, for the pores close to the incidence surface the light field has not a diffuse nature but partially preserves the direction of propagation as well as its polarization and phase [4].

Unusual spectral shapes were also observed in pump-probe spectroscopy performed with two different lasers (tuned around the $\mathrm{D} 1$ transitions of $\mathrm{Rb}$ ) that are essentially due to the diffusive nature of light fields. A simple model neglecting coherent effects and considering completely randomized wave-vectors satisfactorily reproduce the full variety of lineshapes observed [3]. 

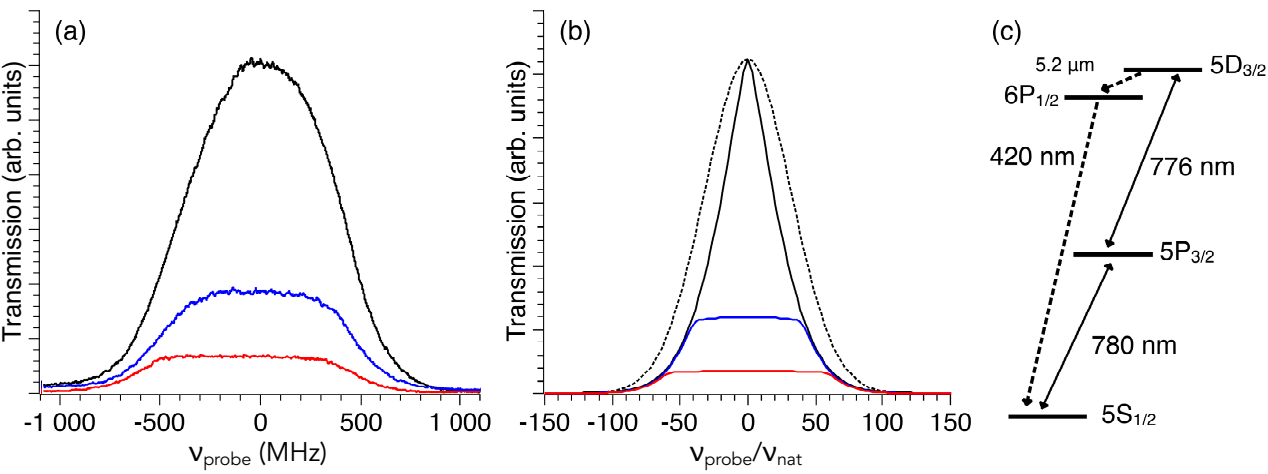

Fig. 1. (Color online) a) Measured spectra scanning the probe laser (D2) around the transition ${ }^{85} \mathrm{Rb}\left(\mathrm{F}=2 \rightarrow \mathrm{F}^{\prime}\right)$ at different frequencies for the coupling laser in $100 \mu \mathrm{m}$ pores. b) Simplified model for the same conditions as a), (not considering the full hyperfine structure of level $5 \mathrm{P}_{3 / 2}$ ). The dashed curve is a Gaussian with Doppler width for comparison.

\section{Highly excited energy levels}

Using two-photon excitation, one can reach atomic states whose lifetime exceeds the mean time of flight of atoms in the pores. The level $5 \mathrm{D}_{3 / 2}$ of $\mathrm{Rb}$ (mean lifetime of around $240 \mathrm{~ns}$ ) was excited using a probe laser resonant with the $\mathrm{D} 2$ line $(780 \mathrm{~nm})$ and a second coupling laser at $776 \mathrm{~nm}$ resonant with the transition $5 \mathrm{P}_{3 / 2} \rightarrow 5 \mathrm{D}_{3 / 2}$. The $5 \mathrm{D}$ level can decay through the $6 \mathrm{P}_{1 / 2}$ level resulting in blue fluorescence at $420 \mathrm{~nm}$ (Fig.1.c). We have detected the blue fluorescence through the porous medium as a measurement of the population of the 5D level. Typical spectra are shown in Fig.1.a. obtained by scanning the frequency of the probe laser (D2), for different frequencies of the coupling laser. Notice that when the coupling laser is resonant, the predicted spectra is narrower than the Doppler width. However, for a detuned coupling laser, the shape of these spectra has two remarkable properties, a flat top and symmetry around resonance.

The symmetry of the spectra can be explained if the contribution of coherent effects are neglected in the two-photon absorption since this results in spectral profiles centred on the atomic transition. Neglecting the coherence contribution is consistent with the phase randomization of the two fields. The flat top of the spectra is also a consequence of the diffuse nature of the optical fields. Satisfactory predictions of the measured spectra shapes (Fig.1.b) is obtained with a simplified model based on the assumptions of two-step (incoherent) two-photon excitation and isotropic distribution of the wave-vectors of the two laser fields.

It is worth mentioning that when the pore dimensions are reduced to about $10 \mu \mathrm{m}$, introducing then a spatial confinement that limits the mean time of flight of atoms to around $50 \mathrm{~ns}$, much shorter than 5D level lifetime, no significant modifications of the spectral shapes were observed in comparison with samples with larger pores ( $\sim 100 \mu \mathrm{m}$ ). This is in contrast with the observation in thin cells with with 1D confinement and light beams propagating perpendicular to the cell windows where a narrowing of the fluorescence spectrum was observed as a consequence of the spatial confinement. For 1D confinement, the contribution of resonant atoms flying nearly parallel to the windows is favored over those that have large velocities in the direction perpendicular to the windows and consequently a short time of flight [5]. We interpret the non observation of such narrowing in the porous material as resulting from the 3D confinement in which the time of flight is mainly determined by the mean velocity modulus which is only loosely selected by the laser frequency (except for very large detunings).

\subsection{Rydberg atoms under $3 D$ confinement}

We are currently exploring the excitation of Rb Rydberg atoms under a 3D confinement in micrometric pores in glass. 1D and 2D confined Rydberg atoms were previously studied in thin cells [6] and in hollow fibers [7]. In our experiment we excite Rydberg atoms using a probe laser resonant with the D1 transition of Rb, and a second coupling 
laser at around $476 \mathrm{~nm}$ that can excite $n S$ and $n D_{3 / 2}$ levels with principal quantum numbers $\mathrm{n}$ ranging from 30 to 40. Both lasers are superposed and focalized to a spot of $50 \mu \mathrm{m}$ in diameter at the porous medium surface, and the backscattered light of the probe field is detected with a photodiode. The probe backscattered light was recorded scanning the coupling laser while keeping the probe laser locked to the D1 transition. Two different porous media were used having $100 \mu \mathrm{m}$ and $10 \mu \mathrm{m}$ pore sizes approximately. The atomic density was approximately $10^{14}$ atomes $/ \mathrm{cm}^{3}$.

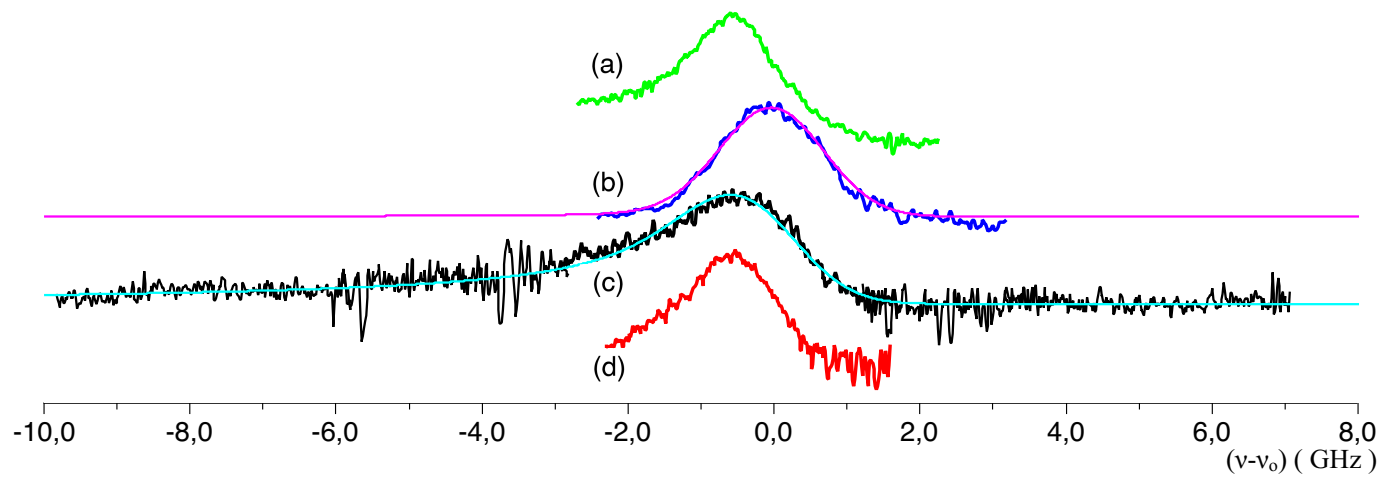

Fig. 2. (Color online) Registered spectra for $100 \mu \mathrm{m}$ pores (a,b), for $10 \mu \mathrm{m}$ pores (c,d) and for different Rydberg levels $33 \mathrm{D}_{3 / 2}(\mathrm{a}), 32 \mathrm{D}_{3 / 2}(\mathrm{~b})$,(c) and $31 \mathrm{D}_{3 / 2}(\mathrm{~d})$. The frequency axis is centered at the resonance frequency of the corresponding level (provided by an external reference). Continuous line: theoretical model.

Some spectra are shown at Fig.2. The spectra recorded for $100 \mu \mathrm{m}$ pores (Fig.2.a,b) have a Doppler width most probably due to the diffuse nature of light. However they have a clear asymmetry that suggest that they are also broadened by the Casimir-Polder interaction of the atoms with the dielectric walls of the pores [6]. This interaction is usually attractive (when it is not a resonant interaction [8]), introducing then a shift in the atomic level to lower frequencies. This suggestion is consistent with the measurement performed for the Rydberg atoms confined to $10 \mu \mathrm{m}$ cavities. The spectrum shown in Fig.2.c for the same Rydberg level as in Fig.2.b has an important increase in the red wing of the spectra. Also shown in Fig-2.b,c is a fit with a theoretical model using the coefficient of a Casimir-Polder atom-surface interaction as the only free parameter. The model predicts a potential corresponding to a $32 \mathrm{D}_{3 / 2}$ level shift of $4 \mathrm{MHz}$ at $10 \mu \mathrm{m}$ distance, a value consistent with a non-resonant interaction. The level dependent interaction is evidenced by comparing spectra Fig.2.c-d. Further work is underway.

\section{References}

1. S. Villalba , H. Failache, A. Laliotis, L. Lenci, S. Barreiro, A. Lezama, "Rb optical resonance in a random porous medium", Optics Letters 38(2), 193-195 (2013).

2. S. Villalba, A. Laliotis, L. Lenci, D. Bloch, A. Lezama, H. Failache, "Sub-Doppler resonances in the backscattered light from random porous media infused with Rb vapor", Phys. Rev. A 89, 023422 (2014).

3. S. Villalba, H. Failache, L. Lenci, A. Lezama, "Nonlinear atomic spectroscopy inside a random porous medium", Phys. Rev. A 90, 052518 (2014).

4. S. Villalba, A. Lezama and H. Failache (unpublished).

5. D. Sarkisyan, D. Bloch, A. Papoyan, M. Dulcoy, "Sub-Doppler spectroscopy by sub-micron thin Cs vapor layer", Opt. Comm. 200, 201-208 (2001).

6. H. Kübler, J. P. Shaffer, T. Baluktsian, R. Löw and T. Pfau, "Coherent excitation of Rydberg atoms in micrometre-sized atomic vapour cells", Nature Photon. 4, 112-116 (2010).

7. G. Epple, K. S. Kleinbach, T. G. Euser, N. Y. Joly, T. Pfau, P. St.J. Russell and R. Löw, "Rydberg atoms in hollow-core photonic crystal fibres", Nature Comm. 5, 1-5 (2014).

8. H. Failache, S. Saltiel, M. Fichet, D. Bloch and M. Ducloy, "Resonant van der Waals repulsion between excited Cs atoms and sapphire surface", Phys. Rev. Lett.83, 5467-5470 (1999). 